DFTT-52/95

hep-th/9509031

August 1995

\title{
FREE DIFFERENTIAL ALGEBRAS: THEIR USE IN FIELD THEORY AND DUAL FORMULATION
}

\author{
Leonardo Castellani \\ II Facoltà di Scienze M.F.N. di Torino, sede di Alessandria \\ Dipartimento di Fisica Teorica \\ and \\ Istituto Nazionale di Fisica Nucleare \\ Via P. Giuria 1, 10125 Torino, Italy.
}

\author{
Alberto Perotto \\ Dipartimento di Fisica Teorica \\ Via P. Giuria 1, 10125 Torino, Italy.
}

\begin{abstract}
The gauging of free differential algebras (FDA's) produces gauge field theories containing antisymmetric tensors. The FDA's extend the CartanMaurer equations of ordinary Lie algebras by incorporating $p$-form potentials $(p>1)$. We study here the algebra of FDA transformations. To every $p$-form in the FDA we associate an extended Lie derivative $\ell$ generating a corresponding "gauge" transformation. The field theory based on the FDA is invariant under these new transformations. This gives geometrical meaning to the antisymmetric tensors. The algebra of Lie derivatives is shown to close and provides the dual formulation of FDA's.
\end{abstract}

e-mail: castellani@to.infn.it

Talk presented by. L. Castellani at the $4^{\text {th }}$ Colloquium "Quantum groups and integrable systems", Prague, June 1995. 


\section{Introduction}

Free differential algebras [1, 2, 3] have emerged as underlying symmetries of field theories containing antisymmetric tensors, as for example supergravity and superstring theories. Within the group-geometric method of ref.s [4, [3, [0], a systematic algorithm exists that produces lagrangians invariant under any given FDA.

Actually, the FDA symmetries related to the antisymmetric tensors were not explicitly discussed in [1, 2, 3]. They were treated in [6, 5], where they could be deduced from the BRST algebra of FDA's by interpreting the ghosts as gauge parameters.

Here we present a direct geometric interpretation of antisymmetric tensors: they are the gauge fields of gauge transformations generated by a new type of Lie derivative. These new Lie derivatives, together with the usual Lie derivatives along the Lie algebra tangent vectors, close on an algebra that can be called the dual formulation of FDA's, extending ordinary Lie algebras.

Our arguments are developed in the case of the simplest FDA extension of the Cartan-Maurer equations, containing a 2-form.

\section{Group geometry and dynamical fields}

We sketch the basic steps of the group-geometric approach of ref.s [ [⿴囗十, [3]. See [5] for a short review.

Lie algebra, tangent vectors, vielbeins

Consider an ordinary Lie algebra $\operatorname{Lie}(G)$, with abstract generators $T_{A}$ satisfying the commutation relations

$$
\left[T_{A}, T_{B}\right]=C^{C}{ }_{A B} T_{C}
$$

On the group manifold $G$ we can find a basis of tangent vectors $\mathbf{t}_{A}$ closing on the same algebra as in (2.1). Their duals are the left-invariant one-forms $\sigma^{A}$ (cotangent basis), also called vielbeins, satisfying the Cartan-Maurer equations:

$$
d \sigma^{A}+\frac{1}{2} C^{A}{ }_{B C} \sigma^{B} \wedge \sigma^{C}=0
$$

as can be seen by using (2.1) and $\sigma^{A}\left(\mathbf{t}_{B}\right)=\delta_{A}^{B}$. Thus, the commutation algebra (2.1) and the Cartan-Maurer equations (2.2) are equivalent descriptions of the same group structure. The Jacobi identities

$$
C^{A}{ }_{B[C} C^{B}{ }_{D E]}=0
$$

necessary for the consistency of (2.1) ensure the integrability of eq.s (2.2), that is the nilpotency of the external derivative $d^{2}=0$. 
Dynamical fields, curvatures and Bianchi identities

The main idea of ref.s [4, 3] is to consider the one-forms $\sigma^{A}$ as the fundamental fields of the geometric theory to be constructed. More precisely, the dynamical fields are the vielbeins $\mu^{A}$ of $\tilde{G}$, a smooth deformation of the group manifold $G$ referred to as "soft group manifold". In general $\mu^{A}$ does not satisfy the Cartan-Maurer equations any more, so that

$$
d \mu^{A}+\frac{1}{2} C^{A}{ }_{B C} \mu^{B} \wedge \mu^{C} \equiv R^{A} \neq 0
$$

The extent of the deformation $G \rightarrow \tilde{G}$ is measured by the curvature two-form $R^{A}$. $R^{A}=0$ implies $\mu^{A}=\sigma^{A}$ and viceversa. The deformation is necessary in order to allow configurations with nonvanishing curvature.

Applying the external derivative $d$ to the definition (2.4), using $d^{2}=0$ and the Jacobi identities (2.3), yields the Bianchi identities

$$
(\nabla R)^{A} \equiv d R^{A}-C_{B C}^{A} R^{B} \wedge \mu^{C}=0
$$

An example: $G=$ Poincaré group

Consider $\tilde{G}=$ smooth deformation of the Poincaré group, whose structure constants are read off the corresponding Lie algebra :

$$
\begin{aligned}
& {\left[P_{a}, P_{b}\right]=0} \\
& {\left[M_{a b}, M_{c d}\right]=\eta_{a d} M_{b c}+\eta_{b c} M_{a d}-\eta_{a c} M_{b d}-\eta_{b d} M_{a c}} \\
& {\left[M_{a b}, P_{c}\right]=\eta_{b c} P_{a}-\eta_{a c} P_{b}}
\end{aligned}
$$

Denoting by $V^{a}$ and $\omega^{a b}$ the vielbein $\mu^{A}$ when the index A runs on the translations and on the Lorentz rotations respectively, eq.s (2.4) take the form:

$$
\begin{aligned}
& R^{a}=d V^{a}-\omega^{a b} \wedge V^{c} \eta_{b c} \\
& R^{a b}=d \omega^{a b}-\omega^{a c} \wedge \omega^{d b} \eta_{c d}
\end{aligned}
$$

The fundamental fields $V^{a}$ and $\omega^{a b}$ are interpreted as the ordinary vierbein and the spin connection, respectively, and eq.s (2.10) define the torsion and the Riemann curvature. These satisfy the Bianchi identities

$$
\begin{aligned}
& d R^{a}-R^{a b} V^{b}+\omega^{a b} R^{b} \equiv \mathcal{D} R^{a}-R^{a b} V^{b}=0 \\
& d R^{a b}-R^{a c} \omega^{c b}+\omega^{a c} R^{c b} \equiv \mathcal{D} R^{a b}=0
\end{aligned}
$$

Products between forms are understood to be exterior products, $\mathcal{D}$ is the Lorentz covariant derivative, and repeated indices are contracted with the Minkowski metric $\eta_{a b}$. 
Note that the fields $\mu^{A}(y)$ depend on all the soft group manifold coordinates $y$. In the Poincaré example, this means that the vierbein and the spin connection depend on the coordinates $y^{a}$ associated to the translations (the ordinary spacetime coordinates) and on the coordinates $y^{a b}$ associated to the Lorentz rotations. Since we want to have space-time fields at the end of the game, we have to find a way to remove the $y^{a b}$ dependence. This is achieved when the curvatures are horizontal in the $y^{a b}$ directions (see later).

How do we find the dynamics of $\mu^{A}(y)$ ? We want to obtain a geometric theory, i.e. invariant under diffeomorphisms of the soft group manifold $\tilde{G}$. We need therefore to construct an action invariant under diffeomorphisms, and this is simply achieved by using only diffeomorphic invariant operations as the exterior derivative and the wedge product. The building blocks are the one-form $\mu^{A}$ and its curvature two-form $R^{A}$, and exterior products of them can make up a lagrangian D-form (where $\mathrm{D}$ is the dimension of space-time).

\section{Diffeomorphisms}

The variation under diffeomorphisms $y+\varepsilon$ of the vielbein field $\mu^{A}(y)$ is given by the Lie derivative of the vielbein along the infinitesimal tangent vector $\epsilon \equiv \varepsilon^{A} \mathbf{t}_{A}$ :

$$
\delta \mu^{A}=\mu^{A}(y+\varepsilon)-\mu^{A}(y)=d\left(i_{\epsilon} \mu^{A}\right)+i_{\epsilon} d \mu^{A} \equiv \ell_{\epsilon} \mu^{A}
$$

On p-forms $\omega_{(p)}=\omega_{B_{1} \ldots B_{p}} \mu^{B_{1}} \wedge \ldots \wedge \mu^{B_{p}}$, the contraction $i_{\mathbf{v}}$ along an arbitrary tangent vector $\mathbf{v}=v^{A} \mathbf{t}_{A}$ is defined as

$$
i_{\mathbf{v}} \omega_{(p)}=p v^{A} \omega_{A B_{2} \ldots B_{p}} \mu^{B_{2}} \wedge \ldots \wedge \mu^{B_{p}}
$$

and maps $p$-forms into $(p-1)$-forms.

The operator

$$
l_{\mathbf{v}} \equiv d i_{\mathbf{v}}+i_{\mathbf{v}} d
$$

is the Lie derivative along the tangent vector $\mathbf{v}$ and maps $p$-forms into $p$-forms. Eq. (2.13) gives the variation under diffeomorphisms of any $p$-form.

We now rewrite the variation $\delta \mu^{A}$ of eq. (2.13) in a suggestive way, by adding and subtracting $C^{A}{ }_{B C} \mu^{B} \varepsilon^{C}$ :

$$
\begin{aligned}
& \delta \mu^{A}=d \varepsilon^{A}+C_{B C}^{A} \mu^{B} \varepsilon^{C}-2 \mu^{B} \varepsilon^{C}\left(d \mu^{A}\right)_{B C}-C_{B C}^{A} \mu^{B} \varepsilon^{C} \\
& =(\nabla \varepsilon)^{A}+i_{\epsilon} R^{A}
\end{aligned}
$$

where we have used the definition (2.4) for the curvature, and the $G$-covariant derivative $\nabla$ acts on $\varepsilon^{A}$ as

$$
(\nabla \varepsilon)^{A} \equiv d \varepsilon^{A}+C_{B C}^{A} \mu^{B} \varepsilon^{A}
$$




\section{Horizontality}

All the invariances of the geometric theory are contained in eq. (2.16). In particular, suppose that the two-form $R^{A}=R_{B C}^{A} \mu^{B} \wedge \mu^{C}$ has vanishing components along the directions of a subgroup $H$ of $G$ :

$$
R_{B H}^{A}=0 \quad \begin{array}{ll}
\text { A runs on } \mathrm{G} \\
\text { H runs on } \mathrm{H}
\end{array}
$$

Then we say that $R^{A}$ is horizontal on $H$, and the diffeomorphisms along the $H$ directions reduce to gauge transformations:

$$
\delta \mu^{A}(y)=(\nabla \varepsilon)^{A}
$$

Moreover, the dependence on the $y^{H}$ coordinates becomes inessential, in the sense that it factorizes after a finite gauge transformation (see ref.s [3, 5]). The theory "remembers" the invariance under $y^{H}$-diffeomorphisms by retaining the gauge invariance under $H$, with $\varepsilon^{H}$ interpreted now as a gauge parameter.

For example, in Poincaré gravity the curvatures are horizontal along the Lorentz directions : then the fields $V^{a}$ and $\omega^{a b}$ live on the coset space

$$
\frac{\mathrm{G}}{\mathrm{H}}=\frac{\text { Poincare }^{\prime}}{\text { Lorentz }}
$$

i.e. on ordinary spacetime. The lagrangian is integrated on a D- volume (Ddimensional spacetime), and is therefore a D-form. The resulting theory is invariant under D-spacetime diffeomorphisms, and under local Lorentz rotations.

Finally, we recall the algebra of Lie derivatives on the group manifold. From

$$
\ell_{\varepsilon^{B} \mathbf{t}_{B}} \mu^{A}=d \varepsilon^{A}+\left(C_{B C}^{A}-2 R_{B C}^{A}\right) \mu^{B} \varepsilon^{C}
$$

cf. (2.16), we deduce

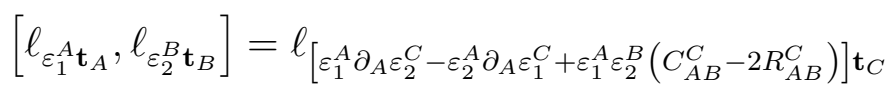

where the partial derivative $\partial_{A}$ of a function $f$ is defined by $d f \equiv\left(\partial_{A} f\right) \mu^{A}$ or also $\partial_{A} f \equiv \mathbf{t}_{A}(f)$. One can can verify the standard formula:

$$
\left[\ell_{\varepsilon_{1}^{A} \mathbf{t}_{A}}, \ell_{\varepsilon_{2}^{B} \mathbf{t}_{B}}\right]=\ell_{\left[\varepsilon_{1}^{A} \mathbf{t}_{A}, \varepsilon_{2}^{B} \mathbf{t}_{B}\right]}
$$

In particular, the Lie derivatives $\ell_{\mathbf{t}_{A}}$ on the undeformed group manifold $G$ close on the Lie algebra:

$$
\left[\ell_{\mathbf{t}_{A}}, \ell_{\mathbf{t}_{B}}\right]=C_{A B}^{C} \ell_{\mathbf{t}_{C}}
$$




\section{$3 \quad$ Free differential algebras}

The dual formulation of Lie algebras provided by the Cartan-Maurer equations (2.2) can be naturally extended to $p$-forms $(p>1)$ :

$$
d \theta_{(p)}^{i}+\sum \frac{1}{n} C_{i_{1} \ldots i_{n}}^{i} \theta_{\left(p_{1}\right)}^{i_{1}} \wedge \ldots \wedge \theta_{\left(p_{n}\right)}^{i_{n}}=0, \quad p+1=p_{1}+\ldots+p_{n}
$$

$p, p_{1}, \ldots p_{n}$ are the degrees of the forms $\theta^{i}, \theta^{i_{1}}, \ldots, \theta^{i_{n}}$; the indices $i, i_{1}, \ldots, i_{n}$ run on irreps of a group $G$, and $C^{i}{ }_{i_{1} \ldots i_{n}}$ are generalized structure constants satisfying generalized Jacobi identities due to $d^{2}=0$. When $p=p_{1}=p_{2}=1$ and $i, i_{1}, i_{2}$ belong to the adjoint representation of $G$, eq.s (6.1) reduce to the ordinary Cartan-Maurer equations. The (anti)symmetry properties of the indices $i_{1}, \ldots i_{n}$ depend on the bosonic or fermionic character of the forms $\theta^{i_{1}}, \ldots \theta^{i_{n}}$

If the generalized Jacobi identities hold, eq.s (3.1) define a free differential algebra (FDA). The possible FDA extensions $G^{\prime}$ of a Lie algebra $G$ have been studied in ref.s [1, 2], and rely on the existence of Chevalley cohomology classes in $G$ [7]. Suppose that, given an ordinary Lie algebra $G$, there exists a $p$-form:

$$
\Omega_{(p)}^{i}(\sigma)=\Omega_{A_{1} \ldots A_{p}} \sigma^{A_{1}} \wedge \ldots \wedge \sigma^{A_{p}}, \quad \Omega_{\mathrm{A}_{1} \ldots \mathrm{A}_{\mathrm{p}}}^{\mathrm{i}}=\text { constants, i runs on a } \mathrm{G} \text { - irrep }
$$

which is covariantly closed but not covariantly exact, i.e.

$$
\nabla \Omega_{(p)}^{i} \equiv d \Omega_{(p)}^{i}+\sigma^{A} \wedge D\left(T_{A}\right)_{j}^{i} \Omega_{(p)}^{j}=0, \quad \Omega_{(p)}^{i} \neq \nabla \Phi_{(p-1)}^{i}
$$

Then $\Omega^{i}{ }_{(p)}$ is said to be a representative of a Chevalley cohomology class in the $D^{i}{ }_{j}$ irrep of $G$. $\nabla$ is the boundary operator satisfying $\nabla^{2}=0$ (it would be proportional to the curvature 2-form on the soft group manifold). The existence of $\Omega^{i}{ }_{(p)}$ allows the extension of the original Lie algebra $G$ to the FDA $G^{\prime}$ :

$$
\begin{aligned}
& d \sigma^{A}+\frac{1}{2} C_{B C}^{A} \sigma^{B} \wedge \sigma^{C}=0 \\
& \nabla \Sigma_{(p-1)}^{i}+\Omega_{(p)}^{i}(\sigma)=0
\end{aligned}
$$

where $\Sigma_{(p-1)}^{i}$ is a new $(p-1)$-form, not contained in $G$. Closure of eq.s $(6.4)$ is due to $\nabla \Omega_{(p)}^{i}=0$.

It is clear that $\Omega_{(p)}^{i}$ differing by exact pieces $\nabla \Phi_{(p-1)}^{i}$ lead to equivalent FDA's, via the redefinition $\Sigma_{(p-1)}^{i} \rightarrow \Sigma_{(p-1)}^{i}+\Phi_{(p-1)}^{i}$. What we are interested in are really nontrivial cohomology classes satisfying eq.s (3.3).

The whole game can be repeated on the free differential algebra $G^{\prime}$ which now contains $\sigma^{A}, \Sigma_{(p-1)}^{i}$. One looks for the existence of polynomials in $\sigma^{A}, \Sigma_{(p-1)}^{i}$

$$
\Omega_{(q)}^{i}(\sigma, \Sigma)=\Omega_{A_{1} \ldots A_{r} i_{1} \ldots i_{s}}^{i} \sigma^{A_{1}} \wedge \ldots \wedge \sigma^{A_{r}} \wedge \Sigma_{(p-1)}^{i_{1}} \wedge \ldots \wedge \Sigma_{(p-1)}^{i_{s}}
$$

satisfying the cohomology conditions (3.3). If such a polynomial exists, the FDA of eq.s (3.4), (3.5) can be further extended to $G^{\prime \prime}$, and so on. 
Note 1: In constructing $D$-dimensional supergravity theories we usually choose as starting point the superPoincaré Lie algebra. The possible $G^{\prime}$ extensions to FDA's depend on the spacetime dimension $D$. For example in $D=11$ there is a cohomology class of the superPoincaré algebra in the identity representation:

$$
\Omega(V, \omega, \psi)=\frac{1}{2} \bar{\psi} \Gamma^{a b} \psi V^{a} V^{b}
$$

where $\psi$ is the gravitino field, dual to the supersymmetry charge; $d \Omega=0$ holds because of the $D=11$ Fierz identity

$$
\bar{\psi} \Gamma^{a b} \psi \bar{\psi} \Gamma^{a} \psi V^{b}=0
$$

This allows the extension of the algebra (3.4) by means of a three -form $A$ :

$$
d A-\Omega(V, \omega, \psi)=0
$$

Note 2: Only nonsemisimple algebras can have FDA extensions in nontrivial $G$ irreps. Indeed a theorem by Chevalley and Eilenberg [7] states that there is no nontrivial cohomology class of $G$ in nontrivial $G$-irreps when $G$ is semisimple.

As we have done in the case of ordinary Lie algebras, we find a dynamical theory based on FDA's by allowing nonvanishing curvatures. This means, for example, that $D=11$ supergravity is based on a deformation of the fields $V, \omega, \psi, A$ such that the superPoincaré curvatures and the $A$-curvature are different from zero. For the geometric construction of the action we refer the reader to refs. [3, 5]. Other theories with antisymmetric tensors have been interpreted as gaugings of free differential algebras: see [3] for a detailed study.

\section{The FDA1 algebra}

We consider here the simplest extension of a Lie algebra, denoted by FDA1:

$$
\begin{aligned}
& d \sigma^{A}+\frac{1}{2} C_{B C}^{A} \sigma^{B} \sigma^{C}=0 \\
& d B^{i}+C^{i}{ }_{A j} \sigma^{A} B^{j}+\frac{1}{6} C^{i}{ }_{A B C} \sigma^{A} \sigma^{B} \sigma^{C} \equiv \nabla B^{i}+\frac{1}{6} C^{i}{ }_{A B C} \sigma^{A} \sigma^{B} \sigma^{C}=0
\end{aligned}
$$

where $B^{i}$ is a two-form in a representation $D^{i}{ }_{j}$ of $G$. The generalized Jacobi identities $\left(d^{2}=0\right)$, besides the usual ones for $C^{A}{ }_{B C}$, are

$$
\begin{gathered}
C_{A j}^{i} C_{B k}^{j}-C_{B j}^{i} C_{A k}^{j}=C_{A B}^{C} C_{C k}^{i}, \quad \text { representation condition } \\
4 C^{j}{ }_{[A B C} C^{i}{ }_{D] j}+6 C_{[A B}^{E} C_{C D] E}^{i}=0, \quad 3-\text { cocycle condition }
\end{gathered}
$$

Eq. (4.3) implies that $\left(C_{A}\right)^{i}{ }_{j} \equiv C^{i}{ }_{A j}$ is a matrix representation of $G$, while eq. (4.4) is just the statement that $C^{i} \equiv C^{i}{ }_{A B C} \sigma^{A} \sigma^{B} \sigma^{C}$ is a 3-cocycle, i.e. $\nabla C^{i}=0$. If 
we allow the left hand sides of eq.s (4.1), (4.2) to be nonvanishing curvatures $R^{A}$, $R^{i}$ respectively, we find the Bianchi identities:

$$
\begin{aligned}
& d R^{A}-C_{B C}^{A} R^{B} \mu^{C}=0 \\
& d R^{i}-C^{i}{ }_{A j} R^{A} B^{j}+C^{i}{ }_{A j} \mu^{A} R^{j}-\frac{1}{2} C^{i}{ }_{A B C} R^{A} \mu^{B} \mu^{C}=0
\end{aligned}
$$

where we use the same symbol $B^{i}$ for the "soft" 2 -form. The curvatures can be expanded on the $\mu^{A}, B^{i}$ basis as

$$
\begin{aligned}
& R^{i}=R_{A B C}^{i} \mu^{A} \mu^{B} \mu^{C}+R_{A j}^{i} \mu^{A} B^{j} \\
& R^{A}=R_{B C}^{A} \mu^{B} \mu^{C}+R_{i}^{A} B^{i}
\end{aligned}
$$

\section{Lie derivatives}

Using the definition (2.15) and the expression of the FDA curvatures, we find the action of the Lie derivative on $B^{i}$ :

$$
\ell_{\varepsilon^{B} t_{B}} B^{i}=\left(R_{A j}^{i}-C_{A j}^{i}\right) \varepsilon^{A} B^{j}+\left(3 R_{A B C}^{i}-\frac{1}{2} C_{A B C}^{i}\right) \varepsilon^{A} \mu^{B} \wedge \mu^{C}
$$

the action on $\mu^{A}$ remaining the one given in (2.21). Here we find something interesting: the Lie derivatives do not close any more as in eq. (2.22). Before computing this modified algebra, let us define

i) a new contraction operator $i_{\varepsilon^{j}} \mathbf{t}_{j}$ by its action on a generic p-form $\omega=$ $\omega_{i_{1} \ldots i_{n} A_{1} \ldots A_{m}} B^{i_{1}} \wedge \ldots B^{i_{n}} \wedge \mu^{A_{1}} \wedge \ldots \mu^{A_{m}}$ as

$$
i_{\varepsilon^{j} \mathbf{t}_{j}} \omega=n \varepsilon^{j} \omega_{j i_{2} \ldots i_{n} A_{1} \ldots A_{m}} B^{i_{2}} \wedge \ldots B^{i_{n}} \wedge \mu^{A_{1}} \wedge \ldots \mu^{A_{m}}
$$

where $\varepsilon^{j}$ is a 1-form. This operator still maps $p$-forms into $(p-1)$-forms. We can also define the contraction $i_{\mathbf{t}_{j}}$, mapping $\mathrm{p}$-forms into $(p-2)$-forms, from

$$
i_{\varepsilon^{j} \mathbf{t}_{j}}=\varepsilon^{j} i_{\mathbf{t}_{j}}
$$

In particular

$$
i_{\mathbf{t}_{j}}\left(B^{i}\right)=\delta_{j}^{i}
$$

so that $\mathbf{t}_{j}$ can be seen as the "tangent vector" dual to $B^{j}$. Note that $i_{\varepsilon^{j} \mathbf{t}_{j}}$ vanishes on $p$-forms that do not contain at least one factor $B^{i}$.

ii) a new Lie derivative given by:

$$
\ell_{\varepsilon^{i} \mathbf{t}_{i}} \equiv i_{\varepsilon^{i} \mathbf{t}_{i}} d+d i_{\varepsilon^{i} \mathbf{t}_{i}}
$$

This new derivative commutes with $d$, satisfies the Leibnitz rule, and acts on the fundamental fields as

$$
\begin{aligned}
& \ell_{\varepsilon^{j} \mathbf{t}_{j}} \mu^{A}=\varepsilon^{j} R^{A} \\
& \ell_{\varepsilon^{j} \mathbf{t}_{j}} B^{i}=d \varepsilon^{i}+\left(C^{i}{ }_{A j}-R_{A j}^{i}\right) \mu^{A} \wedge \varepsilon^{j}
\end{aligned}
$$


Using these new objects, and the Bianchi identities (4.7), (4.8), we can compute the commutator of two "usual" Lie derivatives (acting on $\mu^{A}$ or on $B^{i}$ ) and find:

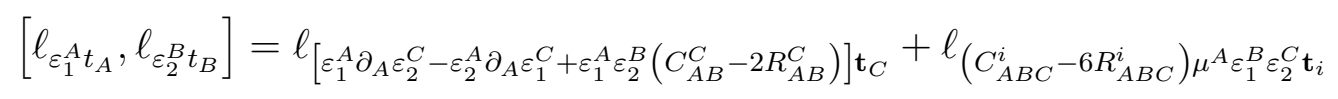

This result has an important consequence: if the field theory based on FDA1 is geometric, i.e. its action is invariant under diffeomorphisms generated by the "usual" Lie derivative, then the new Lie derivative defined in (4.13) must also generate a symmetry of the action, since it appears on the right-hand side of (4.16). Thus, when we construct geometric lagrangians gauging FDA1, we know a priori that the resulting theory will have symmetries generated by the new Lie derivative. In other words, the transformations (4.14), (4.15) are invariances of the action.

It becomes now essential to find the total FDA algebra of transformations, and show that it closes. Using the Bianchi identities, it is a straightforward exercise to find the remaining commutators:

$$
\begin{aligned}
& {\left[\ell_{\varepsilon^{A} \mathbf{t}_{A}}, \ell_{\varepsilon^{j} \mathbf{t}_{j}}\right]=\ell_{\left[\ell_{\varepsilon_{A} \mathbf{t}_{A}} \varepsilon^{k}+\left(C_{B j}^{k}-R_{B j}^{k}\right) \varepsilon^{B} \varepsilon^{j}\right] \mathbf{t}_{k}}} \\
& {\left[\ell_{\varepsilon_{1}^{i} \mathbf{t}_{i}}, \ell_{\varepsilon_{2}^{j} \mathbf{t}_{j}}\right]=\ell_{R_{i}^{B}\left(\varepsilon_{1}^{i}\left(\varepsilon_{2}\right)_{B}^{j}-\varepsilon_{2}^{i}\left(\varepsilon_{1}\right)_{B}^{j}\right) \mathbf{t}_{j}}}
\end{aligned}
$$

where $\varepsilon_{A}^{i}$ are the components of the 1 -form $\varepsilon^{i}$, i.e. $\varepsilon^{i} \equiv \varepsilon_{A}^{i} \mu^{A}$. In particular, we can find the commutators of the Lie derivatives on the rigid FDA1 "manifold" by taking $\varepsilon^{A}=$ const., $\varepsilon_{B}^{i}=$ const. and vanishing curvatures:

$$
\begin{aligned}
& {\left[\ell_{\mathbf{t}_{A}}, \ell_{\mathbf{t}_{B}}\right]=C_{A B}^{C} \ell_{\mathbf{t}_{C}}+C^{i}{ }_{A B C} \ell_{\sigma^{C} \mathbf{t}_{i}}} \\
& {\left[\ell_{\mathbf{t}_{A}}, \ell_{\sigma^{B} \mathbf{t}_{i}}\right]=\left[C^{k}{ }_{A j} \delta_{F}^{B}-\left(C_{A F}^{B}-2 R_{A F}^{B}\right) \delta_{j}^{k}\right] \ell_{\sigma^{F} \mathbf{t}_{k}}} \\
& {\left[\ell_{\sigma^{A} \mathbf{t}_{i}}, \ell_{\sigma^{B} \mathbf{t}_{j}}\right]=0}
\end{aligned}
$$

This algebra can be considered the dual of the FDA1 system given in (4.1), (4.2), and generalizes the Lie algebra of ordinary Lie derivatives (generating usual diffeomorphisms) of (2.24). Notice the essential presence of the 1-form $\sigma$ in front of the "tangent vectors" $\mathbf{t}_{i}$. There are $\operatorname{dim}(G) \times \operatorname{dim}(D)$ independent extended Lie derivatives $\ell_{\sigma^{A} \mathbf{t}_{i}}$, with $\operatorname{dim}(G)=$ dimension of the Lie algebra, $\operatorname{dim}(D)=\operatorname{dimension}$ of the $D^{i}{ }_{j}$ irrep of $G$ to which $B^{i}$ belongs .

\section{References}

[1] D. Sullivan, Infinitesimal computations in topology, Bull. de L' Institut des Hautes Etudes Scientifiques, Publ. Math. 47 (1977).

[2] R. D' Auria and P. Fré, Nucl. Phys. B201 (1982) 101; L. Castellani, P. Fré, F. Giani, K. Pilch and P. van Nieuwenhuizen, Ann. Phys. 146 (1983) 35; P. van Nieuwenhuizen, Free graded differential algebras in: Group theoretical methods in physics, Lect. Notes in Phys. 180 (Springer, Berlin, 1983). 
[3] L. Castellani, R. D'Auria and P. Fré, "Supergravity and Superstrings: a geometric perspective", World Scientific, Singapore 1991.

[4] Y. Ne'eman and T. Regge, Phys. Lett. B74 (1978) 31; Riv. Nuovo Cimento 1 (1978) 5; A. D' Adda, R. D' Auria, P. Fré and T. Regge, Riv. Nuovo Cimento 3 (1980) 6; R. D' Auria, P. Fré and T. Regge, Riv. Nuovo Cimento 3 (1980) 12.

[5] L. Castellani, Int. J. Mod. Phys. A7 (1992) 1583.

[6] S. Boukraa, Nucl. Phys. B303 (1988) 237.

[7] C. Chevalley and S. Eilenberg, Trans. Amer. Math. Soc. 63 (1948) 85. 